\title{
DRAINAGE PATHWAYS OF PULMONARY VEINS IN ATRIAL SEPTAL DEFECT
}

\author{
BY \\ EUGENE BRAUNWALD, CARLOS R. LOMBARDO, AND ANDREW G. MORROW \\ From the Clinic of Surgery, National Heart Institute, Bethesda, Maryland, U.S.A.
}

Received July 20, 1959

At the present time a variety of methods is available for the closure of atrial septal defects; these include closed techniques, semi-open procedures utilizing an atrial well, and open repair with the aid of either general hypothermia or extracorporeal circulation. Since approximately 15 to 20 per cent of patients with atrial septal defect have associated partial anomalous pulmonary venous drainage into the superior vena cava or right atrium (Kirklin et al., 1956 and Gilman et al., 1957), its pre-operative recognition is desirable in the intelligent selection of patients for operation and in planning the surgical procedure itself. Detailed anatomical and physiological assessment is particularly important if extracorporeal circulation is not employed routinely in the closure of atrial septal defects.

The information derived from the clinical examination, electrocardiogram, roentgenogram, angiocardiogram, and usual cardiac catheterization studies has been found to be of relatively little value in determining the site of drainage of pulmonary veins. In the course of the cardiac catheterization, the catheter may frequently be manipulated into one or more pulmonary veins, but even the position of the catheter as determined by fluoroscopic examination is not usually decisive, since it may be impossible to determine whether or not it has traversed the defect before entering the pulmonary vein.

The indicator dilution curve, obtained from a peripheral artery after injection into a pulmonary vein, makes possible the precise determination of the drainage path of the vein. The basis for this diagnostic approach has been described in reports by Swan et al. (1953 and 1957) and Swan and Wood (1957), and from this clinic (Grant et al., 1957; Baker et al., 1958; Braunwald et al., 1958). Following the injection of an indicator into a pulmonary vein that drains normally into the left atrium, the arterial dilution curve closely resembles the curve obtained following left atrial injection, in respect to both appearance time and contour. If, on the other hand, the vein drains into the right atrium or into a systemic vein, the dilution curve will resemble that obtained following right atrial injection. It is the purpose of this communication to present the results of the application of this technique in 29 patients with atrial septal defect in whom the site of drainage of the pulmonary veins was subsequently confirmed at open operation. The operations were performed under direct vision with the aid of extracorporeal circulation in all but two patients in whom general hypothermia was utilized.

\section{MethodS}

The patients studied ranged in age from 3 to 34 years. The presence of a left-to-right shunt entering the right atrium was indicated by a positive nitrous oxide test in each patient (Morrow et al., 1958). Group I consists of 18 patients with ostium secundum atrial defects, normal drainage of all pulmonary veins and only a left-to-right cardiac shunt. Group II consists of 6 patients with interatrial communications with normal drainage of all pulmonary veins but with a significant rightto-left shunt at the atrial level. Included in this group are two patients with uncomplicated ostium 
secundum defects, one with pulmonary stenosis and atrial septal defect, and one with both atrial and ventricular septal defects associated with pulmonary hypertension. One patient had Ebstein's anomaly of the tricuspid valve with a bidirectional atrial shunt and the sixth, previously operated upon at another hospital, had had the inferior vena cava inadvertently diverted into the left atrium when an ostium secundum defect was closed. In Group III are 4 patients who had sinus venosus atrial septal defects with drainage of the right upper and/or right middle lobe pulmonary veins into the superior vena cava and right atrium, but no right-to-left shunt at the atrial level. One patient, Group IV, with a sinus venosus defect and partial anomalous pulmonary venous return as in Group III, had an associated right-to-left shunt at the atrial level.

Dye was injected, as rapidly as possible, into the pulmonary veins which were entered at the time of right heart catheterization, as well as into each atrium. Either tricarbocyanine dye (Fox et al., 1956) or indigo carmine (Lacy et al., 1955) was employed as the indicator. Dilution curves were recorded by withdrawing systemic arterial blood at a rate of $20 \mathrm{ml}$. a minute through a cuvette densitometer (Gilford et al., 1953) by means of a motor-driven syringe. The volume of the tubing between the arterial needle and cuvette was $0.4 \mathrm{ml}$. and the volume of the cuvette itself was $0.3 \mathrm{ml}$. The response of the densitometer to a sudden change in density is 95 per cent in one second and over 99 per cent in two seconds (Sabiston et al., 1957). Since only the appearance time and contour were analysed, the curves were not calibrated. The appearance times were corrected for the delay between the arterial needle and densitometer.

In several of the patients dye was injected into two or more pulmonary veins. When all of these drained normally (Groups I and II) there were no significant differences in either the contours or appearance times; the averages of the latter are presented below. In Groups III and IV the results presented relate to the indicator dilution curves following injections into the anomalous veins.

\section{RESULTS}

Group I (Atrial septal defect (ASD) with normal pulmonary venous drainage). The dilution curves following left atrial and pulmonary venous injections exhibited relatively rapid appearance times ranging from one to six seconds. The appearance times of dye in the arterial blood following pulmonary vein injection were on average $0.2 \mathrm{sec}$. greater than the appearance times following left atrial injection; the difference in appearance times did not exceed 1.0 sec. (Fig. 1). However, the appearance times following right atrial injection were 2.5 to $6.0 \mathrm{sec}$. longer than following pulmonary vein injections, and the difference in appearance times averaged 3.8 seconds. The dilution curves following both left atrial and pulmonary venous injections were characterized by a distinct interruption of the descending limb, signifying a left-to-right shunt (Callahan et al., 1955; Braunwald et al., 1957; and Swan and Wood, 1957b). Curves obtained after right atrial injection had a more gradual ascending limb, and usually a less distinct interruption of the prolonged descending limbs (Fig. 2 and 3).

Group II (ASD, bidirectional or right-to-left shunt, normal pulmonary venous drainage). The appearance times of dye in the arterial blood following pulmonary vein injection exceeded the appearance times following left atrial injection by an average of $0.5 \mathrm{sec}$. with a maximum difference of 1.6 seconds. In contrast to the patients in Group I, in these patients with associated right-to-left shunts, the appearance times were similar following right atrial and pulmonary venous injections; the average difference was $0.3 \mathrm{sec}$. and the maximum difference was $1.5 \mathrm{sec}$. (Fig. 4). However, the contours of these curves differed strikingly. Right atrial injections resulted in curves with an early initial peak representing that dye that shunted from right to left; this initial deflection was followed by the peak representing the dye that had taken the normal path through the pulmonary circulation. This in turn was followed by the prolonged descending limb indicative of the left-to-right shunt. In contrast, the curves recorded after left atrial and pulmonary venous injections resembled those obtained from patients in Group I and had a large initial peak followed by an interruption of the descending limb (Fig. 2 and 5).

Group III (Sinus venosus ASD, anomalous drainage of right pulmonary veins). When dye was injected into the left atrium it appeared in the peripheral artery 3.0 to $4.3 \mathrm{sec}$. earlier (average $3.6 \mathrm{sec}$.) than following injections into the anomalous pulmonary vein. In contrast with the findings in Group I the appearance times following injection into the anomalously draining pulmonary vein differed less than $1.5 \mathrm{sec}$. from those 


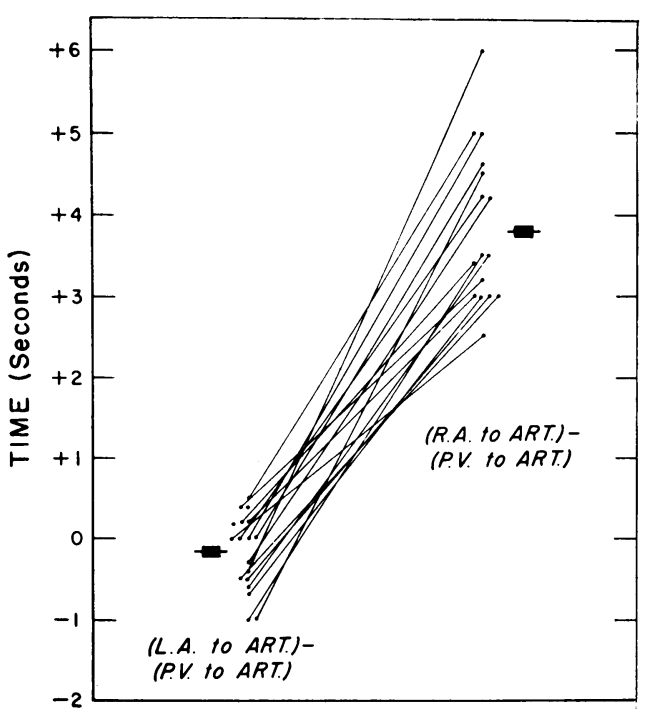

FIG. 1.-Difference in the appearance times of dye in the arterial blood following left atrial (L.A. to Art.) and pulmonary vein (P.V. to Art.) injections and of right atrial (R.A. to Art.) and pulmonary vein (P.V. to Art.) injections in patients in Group I, with ASD and normal pulmonary venous drainage. Solid lines connect observations on one patient and horizontal bars represent the average of appearance time differences.

$$
\text { A.S.D }
$$

L.B. $01-85-76$

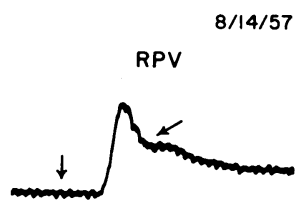

LA

LV

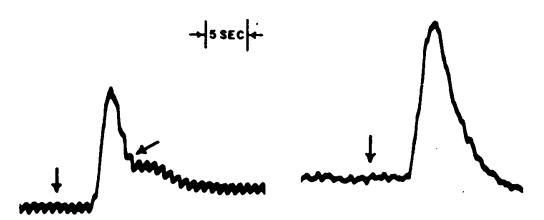

FIG. 3.-Arterial dilution curves recorded in a patient with an atrial septal defect and normal pulmonary venous drainage (Group I). Vertical arrows indicate the time of injection, corrected for the delay in transit between arterial needle and cuvette. Oblique arrows indicate the interruptions of the descending limb of the dilution curve by dye which has been shunted through the pulmonary bed. The injection sites are represented as follows: $\mathrm{RA}=$ right atrium, $\mathrm{RPV}=$ right pulmonary vein, $\mathrm{LA}=$ left atrium, $\mathrm{LV}=$ left ventricle. Note the resemblance in both contour and appearance times between the pulmonary vein and left atrial curves.

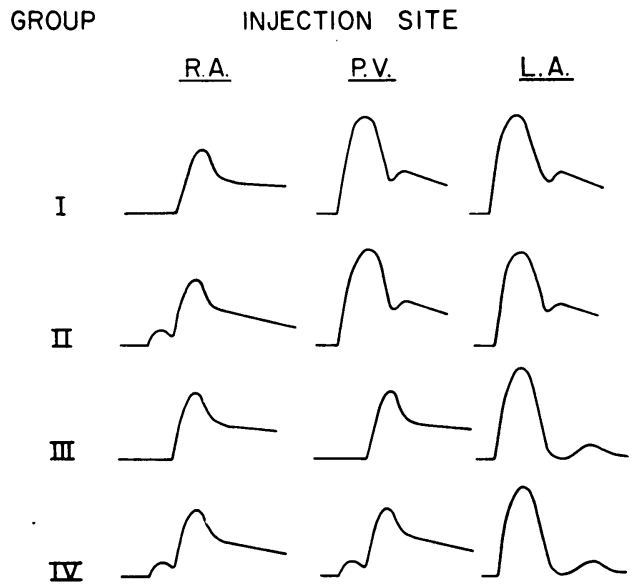

FIG. 2.-Schematic drawings of arterial indicator dilution curves following right atrial (RA), pulmonary vein (PV) and left atrial (LA) injections. The injection time is represented by the beginning of the baseline.

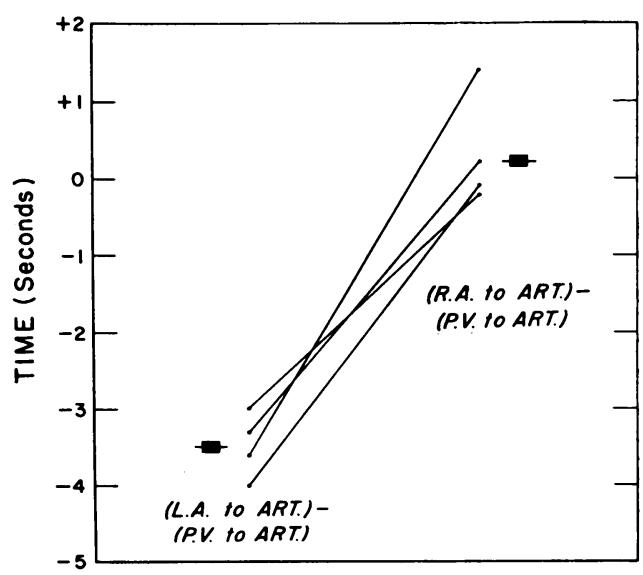

FIG. 4.-Difference in the appearance times of dye in the arterial blood following left atrial and pul. monary vein injections (left) and of right atria and pulmonary vein injections (right) of patients in Group II, with atrial septal defect, bidirectiona or right-to-left shunt and normal pulmonary venous drainage. 


\section{EBSTEIN'S ANOMALY}

L.S. $00-92-65$

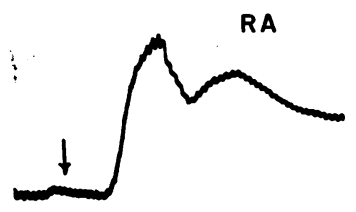

LA
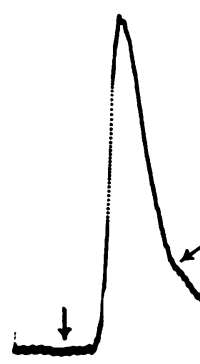

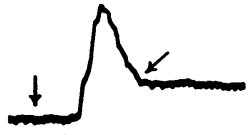

LV

FIG. 5.-Arterial dilution curves recorded in a patient with Ebstein's anomaly of the tricuspid valve, a bidirectional shunt across the interatrial communication and normal pulmonary venous drainage (Group II). Vertical arrows indicate the time of injection, corrected for the delay in transit between arterial needle and cuvette. Oblique arrows indicate the interruption of the descending limb of the dilution curve by dye which has been shunted through the pulmonary bed: The injection sites are represented as follows: $\mathbf{R A}=$ right atrium, $\mathbf{R P V}=$ right pulmonary vein, $\mathrm{LA}=$ left atrium, $\mathrm{LV}=$ left ventricle. All four appearance times are similar but the pulmonary venous curve resembles the left more than the right atrial curve.

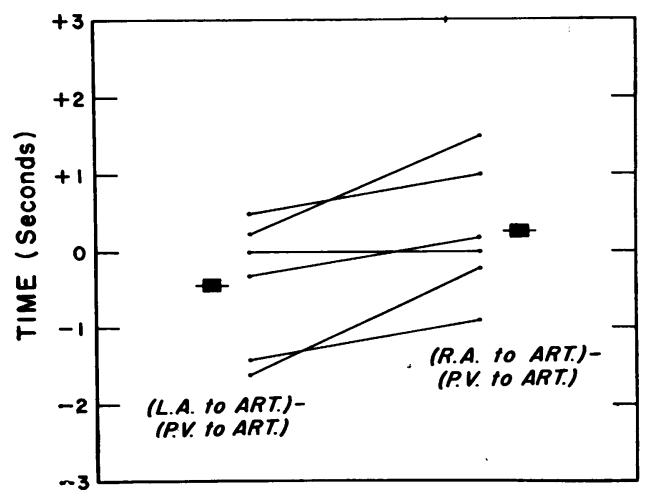

Fig. 6.-Difference in the appearance times of dye in the arterial blood following left atrial and pulmonary vein injections (left) and of right atrial and pulmonary vein injections (right) of patients in Group III, with sinus venosus atrial septal defect and anomalous drainage of right pulmonary veins.

following right atrial injection; the average difference was only $0.3 \mathrm{sec}$. (Fig. 6). In contrast with the findings in Groups I and II the contours of the dilution curves resulting from injections into the anomalous vein were identical with those obtained following right atrial injection (Fig. 2). In none of the four patients in this group could a left-to-right shunt be demonstrated in the dye curves recorded after left atrial injection, and in three of them there was no evidence of a left-to-right shunt from the normally draining left pulmonary veins. These observations suggest that in this group of patients with sinus venosus atrial septal defect the major or entire left-to-right shunt is derived from the anomalous drainage of the pulmonary veins.

Group IV (Sinus venosus ASD, bidirectional shunt, anomalous drainage of right pulmonary veins). In this patient with anomalous pulmonary venous drainage and a right-to-left shunt at the atrial levet, the appearance time following injection into the anomalous vein was similar to that following both right and left atrial injection, as in Group II. However, in contrast to the findings in Group II the contours of the dilution curves following right atrial and anomalous pulmonary vein injection were identical; there was a small early initial peak followed by the larger peak representing dye which had coursed through the pulmonary vascular bed. In this patient no left-to-right shunt was apparent in the dilution curves recorded after left atrial injection (Fig. 2).

\section{Discussion}

When the results of the clinical examinations suggest the presence of some form of atrial septal defect, the catheterization in this clinic is always performed from a saphenous rather than an antecubital vein. With this approach the defect, if present, can be traversed and both right and left 
pulmonary veins can usually be entered to permit the determination of their drainage pathways, by the method described. It is apparent that the drainage pathway of blood from a pulmonary vein is determined primarily by its anatomical relation to the remnant of the interatrial septum and to the defect. When the septum is largely absent posteriorly, as is common with large defects, it may be difficult, even at operation, to decide into which atrium a given vein may have drained primarily. In these circumstances, however, the septum must ordinarily be reconstituted with a prosthesis and this can be placed to the right of the entrance of all pulmonary veins.

When true anomalous drainage into the superior cava and right atrium is present the interatrial communication is generally small and is located high in the septum adjacent to the entrance of the superior vena cava. The complete correction of this combination of anomalies requires the placement of a prosthesis that directs the return from the pulmonary veins through the defect and excludes both from the right atrium and vena cava (Fig. 7). The recognition of this variety of atrial septal defect is particularly important since its complete repair necessitates a prolonged period of circulatory interruption and is not possible by a closed method or during the brief period of cardiotomy possible with hypothermia. Closure of the interatrial defect alone accomplishes little, since all or a major part of the shunt is from the pulmonary veins. Lobectomy, which has been an alternative method of treatment of this lesion (Ehrenhaft et al., 1958) is certainly undesirable.

The indicator dilution method is not only of distinct value in the study of patients with partial anomalous pulmonary venous drainage as described herein, but may also provide the definitive diagnosis of total anomalous pulmonary venous return. In such patients the dilution curves resemble

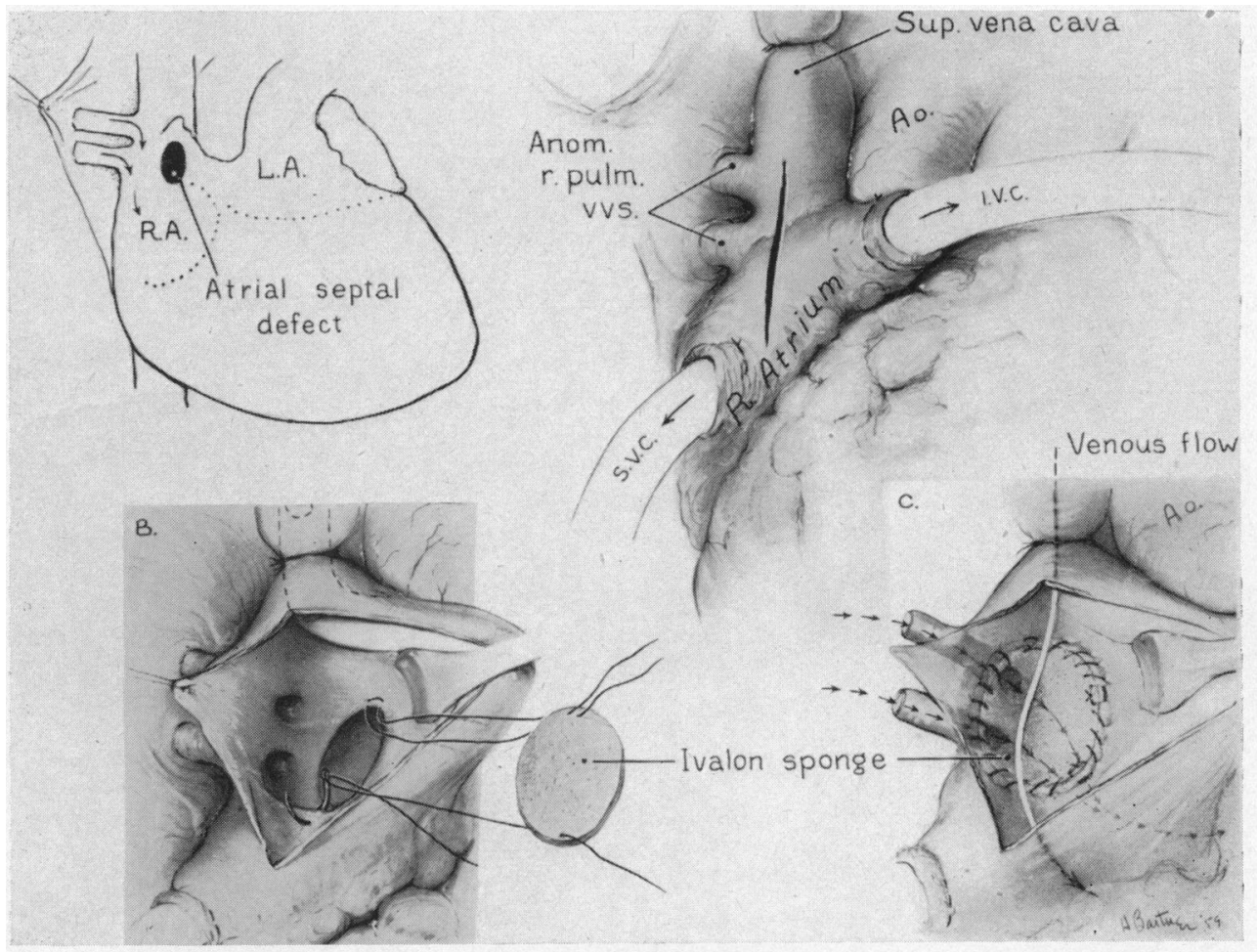

FIG. 7.-Drawings showing method of repair of sinus venosus atrial septal defect with anomalous drainage of two right pulmonary veins. Upper right: An incision is made in the right atrium and superior vena cava after the cavæ have been cannulated and bypass established. Lower left: The atrium and superior vena cava have been opened and the Ivalon sponge prosthesis is sewn into place. Lower right: The prosthetic sponge directs blood from the pulmonary veins across the defect into the left atrium and also prevents shunting of blood from the left atrium into the right. 
those of the patient in Group IV, but the right-to-left shunt is, of course, far greater and is demonstrable following injection into each pulmonary vein. Aside from patients with total anomalous pulmonary venous drainage, we have not encountered a patient with anomalous entrance of a left pulmonary vein into the right atrium or systemic venous bed. However, complete drainage of blood from a left pulmonary vein into the right atrium has been detected in one patient by the indicator dilution method: in this patient at operation the entrance of the left pulmonary veins was found near, but to the left of, the interatrial defect.

\section{SUMMARY}

The paths of pulmonary venous drainage were determined by the indicator dilution method in 29 patients with atrial septal defect who were subsequently operated upon by the open method. The appearance times and contour of the dilution curve, recorded from a systemic artery following injection into the pulmonary vein, were compared with those that resulted from injections into each atrium.

When the curve following pulmonary venous injection closely resembled that following left atrial injection and was dissimilar to that resulting from right atrial injection, anomalous drainage of the vein could be excluded. When the curve closely resembled that following right atrial, but not left atrial injection, anomalous drainage into the right atrium or vena cava was found to be present. The usefulness of these studies in the selection of patients for operation and in the choice of surgical technique is discussed.

\section{REFERENCES}

Baker, R. R., Braunwald, E., Tanenbaum, H. L., and Morrow, A. G. (1958). Ann. Surg., 147, 191.

Braunwald, E., Tanenbaum, H. L., and Morrow, A. G. (1958). Amer. J. Med., 24, 203.

- (1957). Proc. Soc. exp. Biol. N.Y., 94, 510.

Callahan, J. A., Brandenburg, R. O., and Swan, H. J. C. (1955). Circulation, 12, 994.

Ehrenhaft, J. L., Theiler, E. O., and Lawrence, M. S. (1958). Ann. Surg., 148, 249.

Fox, I. J., Brooker, L. G. S., Heseltine, D. W., and Wood, E. H. (1956). Circulation, 14, 937.

Gilford, S. R., Gregg, D. E. Shadle, O. W., Ferguson, T. B., and Marzetta, L. A. (1953). Rev. Sci. Instrum., $24,696$. Gilman, A., Skowron, C. A. R., Musser, B. G., and Bailey, C. P. (1957). Amer. J. Surg., 94, 688.

Grant, R. P., Sanders, R. J., Morrow, A. G., and Braunwald, E. (1957). Circulation, 16, 791.

Kirklin, J. W., Ellis, H. F., Jr., and Wood, E. H. (1956). Surgery, 39, 389.

Lacy, W. W., Ugaz, C., and Newman, E. V. (1955). Circulation Res., 3, 570.

Morrow, A. G., Sanders, R. J., and Braunwald, E. (1958). Circulation, 17, 284.

Sabiston, D. C., Jr., Khouri, E. M., and Gregg, D. E. (1957). Circulation Res., 5, 125.

Swan, H. J. C., Burchell, H. B., and Wood, E. H. (1953). Proc. Mayo Clin., 28, 452.

一, Kirklin, J. W., Becu, L. M., and Wood, E. H. (1957). Circulation, 16, 54.

-, and Wood, E. H. (1957a). Proc. Mayo Clin., 32, 496.

, - - (1957b). Proc. Mayo Clin., 32, 486. 